\title{
Stationary probability distribution of a system with $N$ equal customers with bursty demands connected to a single buffer
}

\author{
R. D. Rodríguez-Said, A. A. Pogorui, and R. M. Rodríguez-Dagnino \\ Communicated by A. Turbin
}

\begin{abstract}
In this paper we study the stationary probability distribution of a system consisting of a finite capacity buffer connected to $N$ equal customers with bursty on-off demands. We assume that the buffer is filled up at a constant rate and we analyze the case when this filling rate satisfies an optimization condition according to the customer demands. First, we consider semi-Markov on-off demands for the case $N=2$ and we model the dynamics of the system using a semi-Markov evolution environment. We show that we can use the phase merging algorithm to reduce the problem to a Markov evolution environment case. Then, we generalize the results for any $N$ using a birth-and-death process.
\end{abstract}

Key words. Random evolutions; stationary distribution; birth-and-death process; Markov bursty demands.

AMS classification. 60K20, 68M10, 90B15, 90B18, 90B30.

\section{Introduction}

The availability of information or product for the supply of different customer demands is a problem that normally involves a buffer (reservoir or container). It is desirable to optimize the amount of stored information according to the expected customer needs and to the amount of incoming information or product feeding into the buffer from the supply line. We will model this system to find the stationary probability distribution of the amount of information stored in the buffer at any time.

In this paper we consider the case of an information server with a single buffer being filled at a constant rate while $N$ equal customers are connected to it. The buffer has finite capacity and the customers demand product or information in a random alternating manner, i.e., they switch from the active (or on) state to the inactive (or off) state. First, we will assume that the alternating demands can be modeled by a semi-Markov stochastic process for the case $N=2$. Then, we will show that we can reduce the semi-Markov process to a Markov process by lumping states according to the phase merging algorithm [2,3]. We continue finding the stationary probability distribution of the system considering a Markov evolution environment and a birthand-death process.

During the active state, each customer demands information at a rate $f$. Hence, when $n$ customers are active, information is demanded at a rate $n \times f$. On the other hand, an unproductive situation is considered if the buffer is empty, i.e., $v=0$. No 
product is required when all customers are inactive. The filling rate of the buffer $F$ is considered a constant. The buffer is filled as long as the amount of information is below its maximum capacity $V$, see Figure 1 .

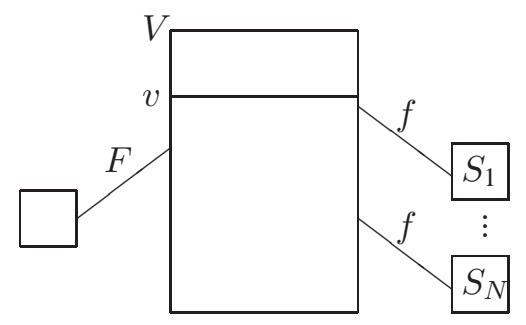

Figure 1. A system of $N$ independent customers and one buffer filled up at a constant rate $F$.

The dynamics of this system can be captured by a first order differential equation that has a random component or the so-called random evolution process [4]. In Section 2 we elaborate our semi-Markov mathematical modeling for the case $N=2$ as an intermediate step to obtain the general solution for any number $N$ of customers. In Section 3 we elaborate our Markov mathematical modeling for any number $N$ of customers and we find a set of recursive equations. In Section 4 we solve the recursive equations to find the stationary probability distribution for $N$ customers.

\section{Mathematical model for $N=2$}

We begin studying the case $N=2$. Consider the semi-Markov process $\{\chi(t)\}$ which is the superposition of two independent alternating semi-Markov processes with the phase space $\mathbb{Z}=\left\{\left(h, x^{i}\right): h \in \mathbb{H}, x^{i} \in \mathbb{R}_{+}^{(2)}\right\}$, where $\mathbb{H}=\left\{h: h=\left(h_{1}, h_{2}\right)\right.$, $\left.h_{i}=0,1 ; i=1,2\right\}$, and $\mathbb{R}_{+}^{2}=\{\vec{x}: \vec{x}=(x, 0), x \geq 0\} \bigcup\{\vec{x}: \vec{x}=(0, x), x \geq 0\}$. We have defined $h_{i}$ as

$$
h_{i}=\left\{\begin{array}{rr}
1, & \text { if } S_{i} \text { is active } \\
0, & \text { if } S_{i} \text { is not active }
\end{array}\right.
$$

where $S_{i}$ stands for subsystem $i$. The component $x$ of the vector $(x, 0)$ (respectively $(0, x)$ ) is the residual life from the last state change of $S_{1}$ (respectively $S_{2}$ ). The initial distribution of $\chi(t)$ is $P\{\chi(0)=(1,1 ; 0,0)\}=1$.

Let us write this in more detail:

$(1,1 ; 0, x)$ - subsystem $S_{1}$ starts to be active and subsystem $S_{2}$ has been active for the time $x$, 
$(1,1 ; x, 0)$ - subsystem $S_{2}$ starts to be active and subsystem $S_{1}$ has been active for the time $x$,

$(1,0 ; 0, x)$ - subsystem $S_{1}$ starts to be active and subsystem $S_{2}$ has been inactive for the time $x$,

$(1,0 ; x, 0)$ - subsystem $S_{2}$ starts to be inactive and subsystem $S_{1}$ has been active for the time $x$,

$(0,1 ; 0, x)$ - subsystem $S_{1}$ starts to be inactive and subsystem $S_{2}$ has been active for the time $x$,

$(0,1 ; x, 0)$ - subsystem $S_{2}$ starts to be active and subsystem $S_{1}$ has been inactive for the time $x$,

$(0,0 ; 0, x)$ - subsystem $S_{1}$ starts to be inactive and subsystem $S_{2}$ has been inactive for the time $x$,

$(0,0 ; x, 0)$ - subsystem $S_{2}$ starts to be inactive and subsystem $S_{1}$ has been inactive for the time $x$.

The embedded Markov chain of this semi-Markov process has the following transition probabilities [2]:

$$
\begin{aligned}
& \mathbf{P}\left[\left(h_{1}, h_{2} ; 0, x\right),\left\{\left(\bar{h}_{1}, h_{2} ; 0, u\right), u \leq y\right\}\right]=\frac{1}{\bar{F}_{h_{2}}^{(2)}(x)} \int_{0}^{y-x} \bar{F}_{h_{2}}^{(2)}(x+u) \mathrm{d} F_{h_{1}}^{(1)}(u), \\
& \mathbf{P}\left[\left(h_{1}, h_{2} ; 0, x\right),\left\{\left(h_{1}, \bar{h}_{2} ; u, 0\right), u \leq y\right\}\right]=\frac{1}{\bar{F}_{h_{2}}^{(2)}(x)} \int_{x}^{y+x} \bar{F}_{h_{1}}^{(1)}(u-x) \mathrm{d} F_{h_{2}}^{(2)}(u), \\
& \mathbf{P}\left[\left(h_{1}, h_{2} ; x, 0\right),\left\{\left(\bar{h}_{1}, h_{2} ; 0, u\right), u \leq y\right\}\right]=\frac{1}{\bar{F}_{h_{1}}^{(1)}(x)} \int_{x}^{y+x} \bar{F}_{h_{2}}^{(2)}(u-x) \mathrm{d} F_{h_{1}}^{(1)}(u), \\
& \mathbf{P}\left[\left(h_{1}, h_{2} ; x, 0\right),\left\{\left(h_{1}, \bar{h}_{2} ; u, 0\right), u \leq y\right\}\right]=\frac{1}{\bar{F}_{h_{1}}^{(1)}(x)} \int_{0}^{y-x} \bar{F}_{h_{1}}^{(1)}(u+x) \mathrm{d} F_{h_{2}}^{(2)}(u),
\end{aligned}
$$

where $\bar{h}_{i}=1-h_{i}, \bar{F}(x)=1-F(x)$, and $F(x)$ is the cumulative distribution function.

The sojourn times corresponding to the stochastic process $\chi(t)$ with phase space $\mathbb{Z}$, have the following expected values

$$
\begin{aligned}
& m\left(h_{1}, h_{2} ; x, 0\right)=\frac{1}{\bar{F}_{h_{1}}^{(1)}(x)} \int_{0}^{\infty} \bar{F}_{h_{1}}^{(1)}(x+y) \bar{F}_{h_{2}}^{(2)}(y) \mathrm{d} y \\
& m\left(h_{1}, h_{2} ; 0, x\right)=\frac{1}{\bar{F}_{h_{2}}^{(2)}(x)} \int_{0}^{\infty} \bar{F}_{h_{1}}^{(1)}(y) \bar{F}_{h_{2}}^{(2)}(x+y) \mathrm{d} y .
\end{aligned}
$$


The density of the stationary distribution for the Markov chain embedded in $\chi(t)$ is of the following form [2]:

$$
\rho\left(h_{1}, h_{2} ; 0, x\right)=c_{s 0} \bar{F}_{h_{2}}^{(2)}(x) \quad \text { and } \quad \rho\left(h_{1}, h_{2} ; x, 0\right)=c_{s 0} \bar{F}_{h_{1}}^{(1)}(x),
$$

where

$$
c_{s 0}^{-1}\left(h_{1}, h_{2}\right)=\int_{0}^{\infty}\left(\bar{F}_{h_{1}}^{(1)}(x)+\bar{F}_{h_{2}}^{(2)}(x)\right) \mathrm{d} x .
$$

Consider a function $C(w)$ on the space $\mathbb{W}=\mathbb{Z} \times[0, V]$ which expresses the rate of change of the amount of information in the buffer and is defined as

$$
C(w)=\left\{\begin{array}{cc}
F, \quad w=\{(0,0 ; \vec{x}), v\},\{\vec{x}=(x, 0)\} \text { or }\{\vec{x}=(0, x)\}, 0<v<V ; \\
F-f, \quad w=\{(0,1 ; \vec{x}), v\},\{\vec{x}=(x, 0)\} \text { or }\{\vec{x}=(0, x)\}, 0<v<V ; \\
F-f, \quad w=\{(1,0 ; \vec{x}), v\},\{\vec{x}=(x, 0)\} \text { or }\{\vec{x}=(0, x)\}, 0<v<V ; \\
F-2 f, \quad w=\{(1,1 ; \vec{x}), v\},\{\vec{x}=(x, 0)\} \text { or }\{\vec{x}=(0, x)\}, 0<v<V ; \\
0, \quad \text { in other cases. }
\end{array}\right.
$$

Let $v(t)$ be the amount of information in the buffer at time $t$. Hence, it is easily verified that $v(t)$ obeys to the differential equation:

$$
\frac{\mathrm{d} v(t)}{\mathrm{d} t}=C(\chi(t), v(t))
$$

with the initial condition $v(0)=v_{0} \in[0, V] . C(w)=C(\chi(t), v(t))$ for $\chi(t) \in \mathbb{Z}$ and $v(t) \in[0, V]$. It can be said that Eq. (2.4) determines the random evolution of the system. Meaning that the process $v(t)$ is the stochastic transfer process in the semi-Markov medium $\chi(t)$ [2], [3]. By using the phase merging algorithm with the merging function $k\left(h_{1}, h_{2} ; \vec{x}\right)=\left(h_{1}, h_{2}\right)$, we can obtain a Markov averaged evolution $\bar{v}(t)$ which is a close approximation to the original semi-Markov case, see Chapter 5 in [3]. Hence the averaged evolution $\bar{v}(t)$ obeys to the following differential equation

$$
\frac{\mathrm{d} \bar{v}(t)}{\mathrm{d} t}=\bar{C}(\bar{\chi}(t), \bar{v}(t)), \bar{v}(0)=\bar{v}_{0} \in[0, V],
$$

where

$$
\bar{C}\left(\left(h_{1}, h_{2}\right), v\right)=\int_{0}^{\infty} C\left\{\left(h_{1}, h_{2} ; \vec{x}\right), v\right\}\left[\rho\left(h_{1}, h_{2} ; 0, x\right)+\rho\left(h_{1}, h_{2} ; x, 0\right)\right] \mathrm{d} x
$$


is a function on $\mathbb{X} \times[0, V]$, where $\mathbb{X}=\{00,01,10,11\}$. The stochastic process $\{\bar{\chi}(t)\}$ is a Markov process with the phase space $\mathbb{X}$. Let us write $\bar{C}\left(\left(h_{1}, h_{2}\right), v\right)$ in more detail,

$$
\begin{gathered}
\bar{C}\left(\left(h_{1}, h_{2}\right), v\right) \\
=\left\{\begin{array}{cc}
F c_{s 0} \int_{0}^{\infty}\left(\bar{F}_{h_{1}}^{(1)}(x)+\bar{F}_{h_{2}}^{(2)}(x)\right) \mathrm{d} x, & \left(h_{1}, h_{2}\right)=(0,0), 0<v<V ; \\
(F-f) c_{s 0} \int_{0}^{\infty}\left(\bar{F}_{h_{1}}^{(1)}(x)+\bar{F}_{h_{2}}^{(2)}(x)\right) \mathrm{d} x, & \left(h_{1}, h_{2}\right)=(1,0), 0<v<V ; \\
(F-f) c_{s 0} \int_{0}^{\infty}\left(\bar{F}_{h_{1}}^{(1)}(x)+\bar{F}_{h_{2}}^{(2)}(x)\right) \mathrm{d} x, & \left(h_{1}, h_{2}\right)=(0,1), 0<v<V ; \\
(F-2 f) c_{s 0} \int_{0}^{\infty}\left(\bar{F}_{h_{1}}^{(1)}(x)+\bar{F}_{h_{2}}^{(2)}(x)\right) \mathrm{d} x, & \left(h_{1}, h_{2}\right)=(1,1), 0<v<V ; \\
0, & \text { in other cases. }
\end{array}\right.
\end{gathered}
$$

From Eqs. (2.1) and (2.2), we can obtain the following equations:

$$
\begin{aligned}
& \mathbf{P}\left\{\left(h_{1}, h_{2}\right)\left(\bar{h}_{1}, h_{2}\right)\right\} \\
= & \frac{1}{c_{s 1}}\left[\int_{0}^{\infty} \rho\left(h_{1}, h_{2} ; 0, x\right) \mathbf{P}\left[\left(h_{1}, h_{2} ; 0, x\right),\left\{\left(\bar{h}_{1}, h_{2} ; 0, u\right)\right\}, u \leq \infty\right] \mathrm{d} x\right. \\
& \left.+\int_{0}^{\infty} \rho\left(h_{1}, h_{2} ; x, 0\right) \mathbf{P}\left[\left(h_{1}, h_{2} ; x, 0\right),\left\{\left(\bar{h}_{1}, h_{2} ; 0, u\right), u \leq \infty\right\}\right] \mathrm{d} x\right], \\
& \mathbf{P}\left\{\left(h_{1}, h_{2}\right)\left(h_{1}, \bar{h}_{2}\right)\right\} \\
= & \frac{1}{c_{s 1}}\left[\int_{0}^{\infty} \rho\left(h_{1}, h_{2} ; 0, x\right) \mathbf{P}\left[\left(h_{1}, h_{2} ; 0, x\right),\left\{\left(h_{1}, \bar{h}_{2} ; 0, u\right)\right\}, u \leq \infty\right] \mathrm{d} x\right. \\
& \left.+\int_{0}^{\infty} \rho\left(h_{1}, h_{2} ; x, 0\right) \mathbf{P}\left[\left(h_{1}, h_{2} ; x, 0\right),\left\{\left(h_{1}, \bar{h}_{2} ; 0, u\right), u \leq \infty\right\}\right] \mathrm{d} x\right],
\end{aligned}
$$

where

$$
\left.c_{s 1}=\int_{0}^{\infty}\left(\rho\left(h_{1}, h_{2} ; 0, x\right)\right)+\rho\left(h_{1}, h_{2} ; x, 0\right)\right) \mathrm{d} x .
$$

The transition probabilities of the corresponding embedded Markov chain can be obtained from Eqs. (2.6) and they are as follows:

$$
\begin{aligned}
& \left.\mathbf{P}\left\{\left(h_{1}, h_{2}\right)\left(\bar{h}_{1}, h_{2}\right)\right)\right\} \\
& =\frac{\int_{0}^{\infty} \int_{0}^{\infty} \bar{F}_{h_{2}}^{(2)}(x+u) \mathrm{d} F_{\bar{h}_{1}}^{(1)}(u) \mathrm{d} x+\int_{0}^{\infty} \int_{0}^{\infty} \bar{F}_{h_{2}}^{(2)}(u) \mathrm{d} u F_{\bar{h}_{1}}^{(1)}(x+u) \mathrm{d} x}{\int_{0}^{\infty} \bar{F}_{h_{1}}^{(1)}(x) \mathrm{d} x+\int_{0}^{\infty} \bar{F}_{h_{2}}^{(1)}(x) \mathrm{d} x},
\end{aligned}
$$




$$
\begin{aligned}
& \left.\mathbf{P}\left\{\left(h_{1}, h_{2}\right)\left(h_{1}, \bar{h}_{2}\right)\right)\right\} \\
& =\frac{\int_{0}^{\infty} \int_{0}^{\infty} \bar{F}_{h_{1}}^{(1)}(x+u) \mathrm{d} F_{\bar{h}_{2}}^{(2)}(u) \mathrm{d} x+\int_{0}^{\infty} \int_{0}^{\infty} \bar{F}_{h_{1}}^{(1)}(u) \mathrm{d} u F_{\bar{h}_{2}}^{(2)}(x+u) \mathrm{d} x}{\int_{0}^{\infty} \bar{F}_{h_{1}}^{(1)}(x) \mathrm{d} x+\int_{0}^{\infty} \bar{F}_{h_{2}}^{(1)}(x) \mathrm{d} x} .
\end{aligned}
$$

The mean sojourn times of the process $\bar{\chi}(t)$ in states from $\mathbb{X}$ are given by

$$
\begin{aligned}
m\left(h_{1}, h_{2}\right)= & \int_{0}^{\infty} \rho\left(h_{1}, h_{2} ; x, 0\right) m\left(h_{1}, h_{2} ; x, 0\right) \mathrm{d} x \\
& +\int_{0}^{\infty} \rho\left(h_{1}, h_{2} ; 0, x\right) m\left(h_{1}, h_{2} ; 0, x\right) \mathrm{d} x \\
= & c_{s 0}\left(\int_{0}^{\infty} \int_{0}^{\infty} \bar{F}_{h_{1}}^{(1)}(y) \bar{F}_{h_{2}}^{(2)}(x+y) \mathrm{d} y \mathrm{~d} x\right. \\
& \left.+\int_{0}^{\infty} \int_{0}^{\infty} \bar{F}_{h_{1}}^{(1)}(x+y) \bar{F}_{h_{2}}^{(2)}(y) \mathrm{d} y \mathrm{~d} x\right) .
\end{aligned}
$$

In summary, by using the merging algorithm, the random evolution $v(t)$ in the semiMarkov medium $\chi(t)$ can be reduced to the Markov evolution $\bar{v}(t)$ in the Markov medium $\bar{\chi}(t)$. So, as an example we consider an evolution in a Markov medium.

Let us introduce the following stochastic process $\{\bar{\chi}(t)\}$ such that

$$
\bar{\chi}(t)= \begin{cases}0, & \text { if no customer is active } \\ 1, & \text { if } S_{1} \text { is active; } \\ 2, & \text { if } S_{2} \text { is active } \\ 3, & \text { if customers } S_{1} \text { and } S_{2} \text { are active; }\end{cases}
$$

The stochastic process $\bar{\chi}$ is a Markov process on the phase space (or states) $\Theta=\{0,1,2,3\}$. Hence, the generating operator (or matrix) of $\bar{\chi}(t)$ can be written as [9]

$$
Q=q[\bar{P}-I]=\left[\begin{array}{cccc}
-2 \lambda & \lambda & \lambda & 0 \\
\mu & -(\lambda+\mu) & 0 & \lambda \\
\mu & 0 & -(\lambda+\mu) & \lambda \\
0 & \mu & \mu & -2 \mu
\end{array}\right],
$$

where $q=\left[q_{i} \delta_{i j} ; i, j \in\{0,1,2,3\}\right]$ is a diagonal matrix of sojourn times intensities of different states and $q_{0}=2 \lambda, q_{1}=\lambda+\mu, q_{2}=\lambda+\mu$ and $q_{3}=2 \mu$. Here, the 
Kronecker delta is defined as

$$
\delta_{i j}= \begin{cases}1, & i=j \\ 0, & i \neq j\end{cases}
$$

We should notice that $q_{\theta}=\left(m\left(h_{1}, h_{2}\right)\right)^{-1}$, with the equivalence

$$
\left(h_{1} h_{2}\right)=\{00,01,10,11\} \Leftrightarrow\{0,1,2,3\}=\Theta \ni \theta .
$$

The elements of the matrix $\bar{P}$ are the transition probabilities of the Markov chain embedded in the Markov process $\bar{\chi}(t)$, i.e.,

$$
\bar{P}=\left[\begin{array}{cccc}
0 & \frac{1}{2} & \frac{1}{2} & 0 \\
\frac{\mu}{\lambda+\mu} & 0 & 0 & \frac{\lambda}{\lambda+\mu} \\
\frac{\mu}{\lambda+\mu} & 0 & 0 & \frac{\lambda}{\lambda+\mu} \\
0 & \frac{1}{2} & \frac{1}{2} & 0
\end{array}\right] .
$$

If we consider that customers $S_{1}$ and $S_{2}$ are equal we can construct a birth-and-death process $\left\{\chi^{(2)}(t)\right\}$ as a simplification of process $\bar{\chi}(t)$.

We consider the superposition of two on-off Markov processes as the birth-anddeath process $\left\{\chi^{(2)}(t)\right\}$ of the following form:

$$
\chi^{(2)}(t)=\left\{\begin{array}{cc}
0, & \text { if no customer is active } \\
1, & \text { if one customer is active } \\
2, & \text { if two customers are active }
\end{array} .\right.
$$

The stochastic process $\chi^{(2)}$ is a Markov process on the phase space (or states) $\Theta^{(2)}=\{0,1,2\}$ with the state diagram shown in Fig. 2.

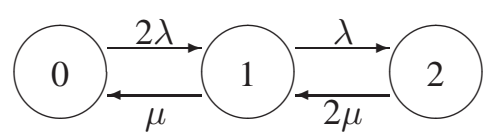

Figure 2. A system of two independent customers that is a birth-and-death process with three states.

Then, for this system we have the following matrix of sojourn time intensities

$$
q^{(2)}=\left[\begin{array}{ccc}
2 \lambda & 0 & 0 \\
0 & \lambda+\mu & 0 \\
0 & 0 & 2 \mu
\end{array}\right]
$$


Also, we have a transition probability matrix given by

$$
P^{(2)}=\left[\begin{array}{ccc}
0 & 1 & 0 \\
\frac{\mu}{\lambda+\mu} & 0 & \frac{\lambda}{\lambda+\mu} \\
0 & 1 & 0
\end{array}\right] \text {. }
$$

Hence, the generating operator (or matrix) can be written as [9]

$$
Q^{(2)}=q^{(2)}\left[P^{(2)}-I\right]=\left[\begin{array}{ccc}
-2 \lambda & 2 \lambda & 0 \\
\mu & -(\lambda+\mu) & \lambda \\
0 & 2 \mu & -2 \mu
\end{array}\right] \text {. }
$$

Then, we consider a function $C^{(2)}(w)$ on the space $\mathbb{W}^{(2)}=\{0,1,2\} \times[0, V]$ defined as

$$
C^{(2)}(w)=\left\{\begin{array}{cc}
F & w=\{0, v\}, 0<v<V ; \\
F-f & w=\{1, v\}, 0<v<V ; \\
F-2 f & w=\{2, v\}, 0<v<V ; \\
0 & \text { other cases }
\end{array} .\right.
$$

Denote as $v(t)$ the amount of information in the buffer at time $t$. It can be easily verified that $v(t)$ satisfies the following equation

$$
\frac{\mathrm{d} v(t)}{\mathrm{d} t}=C^{(2)}\left(\chi^{(2)}(t), v(t)\right)
$$

with the initial condition $v(0)=v_{0} \in[0, V]$. Eq. (2.15) determines the random evolution of the system in the Markov medium $\chi(t)[1]$.

The sojourn time distribution functions, say $F_{\theta}^{(2)}(t)$, have the following form for the different states:

$$
F_{0}^{(2)}(t)=1-e^{-2 \lambda t}, F_{1}^{(2)}(t)=1-e^{-(\lambda+\mu) t} \text {, and } F_{2}^{(2)}(t)=1-e^{-2 \mu t} .
$$

Now, denote as

$$
f_{\theta}^{(2)}=\frac{\mathrm{d} F_{\theta}^{(2)}(t)}{\mathrm{d} t} \text { and } r_{\theta}^{(2)}=\frac{f_{\theta}(t)}{1-F_{\theta}(t)}
$$

for all $\theta \in \Theta^{(2)}$, i.e., $r_{0}^{(2)}=2 \lambda, r_{1}^{(2)}=\lambda+\mu, r_{2}^{(2)}=2 \mu$. Then, $\xi^{(2)}(t)=\left(\chi^{(2)}(t), v(t)\right)$ is a Markov process with the generator $[1,4]$

$$
A^{(2)} \phi(\theta, v)=C^{(2)}(\theta, v) \frac{\partial}{\partial v} \phi(\theta, v)+r_{\theta}^{(2)}\left[P^{(2)} \phi(\theta, v)-\phi(\theta, v)\right]
$$


where

$$
P^{(2)} \phi(\theta, v)=\sum_{y \in \Theta} p_{\theta y} \phi(y, v),
$$

or equivalently,

$$
A^{(2)} \phi(\theta, v)=C^{(2)}(\theta, v) \frac{\partial}{\partial v} \phi(\theta, v)+Q^{(2)} \phi(\theta, v) .
$$

Denote by $\rho$ the stationary distribution of process $\xi^{(2)}(t)$. Then, for every function $\phi(\cdot)$ belonging to the domain of the operator $A$ we have

$$
\int_{\mathbb{W}^{(2)}} A^{(2)} \phi(z) \rho(\mathrm{d} z)=0
$$

The analysis of the properties of process $\xi^{(2)}(t)$ leads up to the conclusion that, for case $f<F<2 f$, the stationary distribution $\rho$ has atoms at points $(2,0),(0, V)$ and $(1, V)$. We denote them as $\rho[2,0], \rho[0, V]$ and $\rho[1, V]$. The continuous part of $\rho$ is denoted by $\rho(\theta, v)$.

Let us write Eq. (2.18) in more detail.

$$
\begin{aligned}
\int_{\mathbb{W}(2)} & A^{(2)} \phi(z) \rho(\mathrm{d} z) \\
= & \int_{0+}^{V-}\left\{\left[F \frac{\partial}{\partial v} \phi(0, v)-2 \lambda \phi(0, v)+2 \lambda \phi(1, v)\right] \rho(0, v)\right. \\
+ & {\left[(F-f) \frac{\partial}{\partial v} \phi(1, v)+\mu \phi(0, v)-(\mu+\lambda) \phi(1, v)+\lambda \phi(2, v)\right] \rho(1, v) } \\
+ & {\left.\left[(F-2 f) \frac{\partial}{\partial v} \phi(2, v)+2 \mu \phi(1, v)-2 \mu \phi(2, v)\right] \rho(2, v)\right\} \mathrm{d} v } \\
+ & {[2 \mu \phi(1,0)-2 \mu \phi(2,0)] \rho[2,0] } \\
+ & {[-2 \lambda \phi(0, V)+2 \lambda \phi(1, V)] \rho[0, V] } \\
+ & {[\mu \phi(0, V)-(\mu+\lambda) \phi(1, V)+\lambda \phi(2, V)] \rho[1, V]=0 . }
\end{aligned}
$$

Let $A^{(2) *}$ be the conjugate operator of $A^{(2)}$. By changing the order of integration in Eq. (2.18) we can obtain the following expression for the continuous part of $A^{(2) *} \rho$.

$$
\left\{\begin{aligned}
-2 \lambda \rho(0, v)+\mu \rho(1, v) & =F \frac{\partial}{\partial v} \rho(0, v) \\
2 \lambda \rho(0, v)-(\mu+\lambda) \rho(1, v)+2 \mu \rho(2, v) & =(F-f) \frac{\partial}{\partial v} \rho(1, v) \\
\lambda \rho(1, v)-2 \mu \rho(2, v) & =(F-2 f) \frac{\partial}{\partial v} \rho(2, v)
\end{aligned}\right.
$$


Eqs. (2.19) can also be stated in the following form:

$$
Q^{(2) T}\left[\begin{array}{l}
\rho(0, v) \\
\rho(1, v) \\
\rho(2, v)
\end{array}\right]=\left[\begin{array}{ccc}
F & 0 & 0 \\
0 & (F-f) & 0 \\
0 & 0 & (F-2 f)
\end{array}\right] \frac{\partial}{\partial v}\left[\begin{array}{c}
\rho(0, v) \\
\rho(1, v) \\
\rho(2, v)
\end{array}\right] .
$$

Even though it is not difficult to find a closed-form solution for this system, we find its solution is an intermediate step to obtain the general solution for any number $N$ of customers. Then, we should generalize the $Q^{(2)}$ matrix.

We obtain the expressions for the atoms for case $f<F<2 f$ as

$$
\left\{\begin{aligned}
-F \rho(0,0+) & =0 \\
-(F-f) \rho(1,0+)+2 \mu \rho[2,0] & =0 \\
-(F-2 f) \rho(2,0+)-2 \mu \rho[2,0] & =0
\end{aligned}\right.
$$

and

$$
\left\{\begin{array}{rl}
F \rho(0, V-)-2 \lambda \rho[0, V]+\mu \rho[1, V] & =0 \\
(F-f) \rho(1, V-)+2 \lambda \rho[0, V]-(\mu+\lambda) \rho[1, V] & =0 \\
(F-2 f) \rho(2, V-)+\lambda \rho[1, V] & =0
\end{array} .\right.
$$

In these equations we have defined the notation

$$
\rho(\theta, 0+):=\lim _{v \downarrow 0} \rho(\theta, v)
$$

and

$$
\rho(\theta, V-):=\lim _{v \uparrow V} \rho(\theta, v) .
$$

It follows from Eqs. (2.19) that

$$
F \rho(0, v)+(F-f) \rho(1, v)+(F-2 f) \rho(2, v)=c=\text { constant }
$$

From Eqs. (2.21) and (2.22) we get $c=0$. Then, we obtain

$$
F \rho(0, v)+(F-f) \rho(1, v)+(F-2 f) \rho(2, v)=0 .
$$

For instance, if we choose $F<f$ instead, we obtain the same result as the one in Eq. (2.26). By analyzing this procedure, it is not difficult to see that this equation can also be generalized to $N$ customers. Also, It should be mentioned that the procedure used to reduce the initial problem to the Markov case is acceptable for any $N$. 


\section{Generalization to $N$ customers}

We consider the superposition of $N$ on-off Markov processes as the birth and death process $\left\{\chi^{(N)}\right\}$ of the following form:

$$
\chi^{(N)}(t)=\left\{\begin{array}{cc}
0, & \text { if no customer is active } \\
1, & \text { if one cutomer is active } \\
2, & \text { if two customers are active } \\
\vdots & \vdots \\
N, & \text { if } N \text { customers are active }
\end{array}\right.
$$

The stochastic process $\chi^{(N)}$ is a Markov process on the phase space (or states) $\Theta^{(N)}=0,1,2, \ldots, N$ with the state diagram shown in Fig. 3.

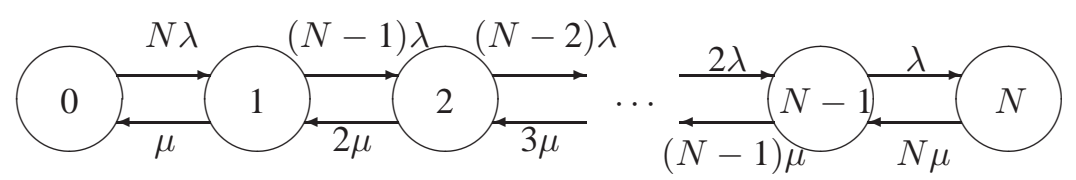

Figure 3. A system of $N$ independent customers that is a birth-and-death process with $N+1$ states.

Then, for this system we have the following matrix of sojourn time intensities

$$
q^{(N)}=\left[\begin{array}{cccccc}
N \lambda & 0 & 0 & \cdots & 0 & 0 \\
0 & \mu+(N-1) \lambda & 0 & \cdots & 0 & 0 \\
0 & 0 & 2 \mu+(N-2) \lambda & \cdots & 0 & 0 \\
\vdots & \vdots & \vdots & \vdots & \vdots & \vdots \\
0 & 0 & 0 & \cdots & (N-1) \mu+\lambda & 0 \\
0 & 0 & 0 & \cdots & 0 & N \mu
\end{array}\right]
$$

Also, we have a transition probability matrix given by 


$$
\begin{aligned}
& P^{(N)}=0 \\
& {\left[\begin{array}{cccccc}
0 & 1 & 0 & \cdots & 0 & 0 \\
\frac{\mu}{\mu+(N-1) \lambda} & 0 & \frac{(N-1) \lambda}{\mu+(N-1) \lambda} & \cdots & 0 & 0 \\
0 & \frac{2 \mu}{2 \mu+(N-2) \lambda} & 0 & \cdots & 0 & 0 \\
\vdots & \vdots & \vdots & \vdots & \vdots & \vdots \\
0 & 0 & 0 & \cdots & 0 & \frac{\lambda}{\lambda+(N-1) \mu} \\
0 & 0 & 0 & \cdots & 1 & 0
\end{array}\right]}
\end{aligned}
$$

Hence, the generating operator (or matrix) can be written as [9]

$$
\begin{aligned}
& Q^{(N)}=q^{(N)}\left[P^{(N)}-I\right]= \\
& {\left[\begin{array}{cccccc}
-N \lambda & N \lambda & 0 & \ldots & 0 & 0 \\
\mu & -N \lambda+\lambda-\mu & (N-1) \lambda & \ldots & 0 & 0 \\
0 & 2 \mu & -N \lambda+2(\lambda-\mu) & \ldots & 0 & 0 \\
\vdots & \vdots & \vdots & \vdots & \vdots & \vdots \\
0 & 0 & 0 & \ldots & 2 \lambda & 0 \\
0 & 0 & 0 & \ldots & -\lambda-(N-1) \mu & \lambda \\
0 & 0 & 0 & \ldots & N \mu & -N \mu
\end{array}\right] .}
\end{aligned}
$$

Then, we consider a function $C^{(N)}(w)$ on the space $\mathbb{W}^{(N)}=\{0,1,2, \ldots, N\} \times$ $[0, V]$ defined as

$$
C^{(N)}(w)=\left\{\begin{array}{cc}
F & w=\{0, v\}, 0<v<V ; \\
F-f & w=\{1, v\}, 0<v<V ; \\
F-2 f & w=\{2, v\}, 0<v<V ; \\
\vdots & \vdots \\
F-(N-1) f & w=\{N-1, v\}, 0<v<V ; \\
F-N f & w=\{N, v\}, 0<v<V ; \\
0 & \text { other cases. }
\end{array}\right.
$$

Denote by $v(t)$ the amount of information in the buffer at time $t$. It can be easily verified that $v(t)$ satisfies the following equation

$$
\frac{\mathrm{d} v(t)}{\mathrm{d} t}=C^{(N)}\left(\chi^{(N)}(t), v(t)\right),
$$


with the initial condition $v(0)=v_{0} \in[0, V]$. Eq. (3.2) determines the random evolution of the system in the Markov medium $\chi^{(N)}(t)[1]$.

The sojourn time probability distribution functions, say $F_{\theta}^{(N)}(t)$, have the following form for the different states:

$$
\left\{\begin{aligned}
F_{0}^{(N)}(t) & =1-e^{-N \lambda t} \\
F_{1}^{(N)}(t) & =1-e^{-((N-1) \lambda+\mu) t} \\
F_{2}^{(N)}(t) & =1-e^{-((N-2) \lambda+2 \mu) t} \\
\vdots & \vdots \\
F_{N-1}^{(N)}(t) & =1-e^{-(\lambda+(N-1) \mu) t} \\
F_{N}^{(N)}(t) & =1-e^{-N \mu t}
\end{aligned}\right.
$$

Now, denote as $f_{\theta}^{(N)}=\frac{\mathrm{d} F_{\theta}}{\mathrm{d} t}$ and $r_{\theta}^{(N)}=\frac{f_{\theta}(t)}{1-F_{\theta}(t)}$ for all $\theta \in \Theta^{(N)}$, i.e.,

$$
\left\{\begin{aligned}
r_{0}^{(N)} & =N \lambda \\
r_{1}^{(N)} & =(N-1) \lambda+\mu \\
r_{2}^{(N)} & =(N-2) \lambda+2 \mu \\
\vdots & \vdots \\
r_{N-1}^{(N)} & =\lambda+(N-1) \mu \\
r_{N}^{(N)} & =N \mu
\end{aligned}\right.
$$

Then, $\xi^{(N)}(t)=\left(\chi^{(N)}(t), v(t)\right)$ is a Markov process with generator [1, 4]

$$
A^{(N)} \phi(\theta, v)=C^{(N)}(\theta, v) \frac{\partial}{\partial v} \phi(\theta, v)+r_{\theta}^{(N)}\left[P^{(N)} \phi(\theta, v)-\phi(\theta, v)\right],
$$

where $P^{(N)} \phi(\theta, v)=\sum_{y \in \Theta} p_{\theta y} \phi(y, v)$, or equivalently,

$$
A^{(N)} \phi(\theta, v)=C^{(N)}(\theta, v) \frac{\partial}{\partial v} \phi(\theta, v)+Q^{(N)} \phi(\theta, v) .
$$

Denote by $\rho$ the stationary distribution of the process $\xi^{(N)}(t)$. Then, for every function $\phi(\cdot)$ belonging to the domain of the operator $A$ we have

$$
\int_{\mathbb{W}^{(N)}} A^{(N)} \phi(z) \rho(d z)=0 .
$$

Let $A^{(N) *}$ be the conjugate operator of $A^{(N)}$. By changing the order of integration in Eq. (3.4) we can obtain the following expression for the continuous part of $A^{(N) *} \rho$. 
$Q^{(N) T} \rho^{(N)}=\left[\begin{array}{cccccc}F & 0 & 0 & \ldots & 0 & 0 \\ 0 & F-f & 0 & \ldots & 0 & 0 \\ 0 & 0 & F-2 f & \ldots & 0 & 0 \\ \vdots & \vdots & \vdots & \vdots & \vdots & \vdots \\ 0 & 0 & 0 & \ldots & 0 & 0 \\ 0 & 0 & 0 & \ldots & F-(N-1) f & 0 \\ 0 & 0 & 0 & \ldots & 0 & F-N f\end{array}\right] \frac{\partial}{\partial v} \rho^{(N)}$

where $\rho^{(N)}=\left[\begin{array}{c}\rho(0, v) \\ \rho(1, v) \\ \rho(2, v) \\ \vdots \\ \rho(N-2, v) \\ \rho(N-1, v) \\ \rho(N, v)\end{array}\right]$ and $\rho(\theta, v)$ denote the continuous part of $\rho$.

Let us write Eqs. (3.5) in more detail.

$$
\begin{aligned}
-N \lambda \rho(0, v)+\mu \rho(1, v) & =F \frac{\partial}{\partial v} \rho(0, v) \\
N \lambda \rho(0, v)-[(N-1) \lambda+\mu] \rho(1, v)+2 \mu \rho(2, v) & =(F-f) \frac{\partial}{\partial v} \rho(1, v) \\
(N-1) \lambda \rho(1, v)-[(N-2) \lambda+2 \mu] \rho(2, v)+3 \mu \rho(3, v) & =(F-2 f) \frac{\partial}{\partial v} \rho(2, v) \\
(N-2) \lambda \rho(2, v)-[(N-3) \lambda+3 \mu] \rho(3, v)+4 \mu \rho(4, v) & =(F-3 f) \frac{\partial}{\partial v} \rho(3, v) \\
\vdots & \vdots \\
\lambda \rho(N-1, v)-N \mu \rho(N, v) & =(F-N f) \frac{\partial}{\partial v} \rho(N, v) .
\end{aligned}
$$

Then, we can write a general equation for any $n$ as

$$
\begin{aligned}
(F-(n-1) f) \frac{\partial}{\partial v} \rho(n-1, v)= & (N-(n-2)) \lambda \rho(n-2, v) \\
& -[(N-(n-1)) \lambda+(n-1) \mu] \rho(n-1, v) \\
& +n \mu \rho(n, v),
\end{aligned}
$$

such that $1<n \leq N$. 
Solving for $\rho(n, v)$ we have

$$
\begin{aligned}
\rho(n, v)= & \frac{(F-(n-1) f)}{n \mu} \frac{\partial}{\partial v} \rho(n-1, v) \\
& +\frac{[(N-(n-1)) \lambda+(n-1) \mu]}{n \mu} \rho(n-1, v) \\
& -\frac{(N-(n-2)) \lambda}{n \mu} \rho(n-2, v),
\end{aligned}
$$

for $1<n \leq N$. We also know that

$$
\rho(1, v)=\frac{F}{\mu} \frac{\partial}{\partial v} \rho(0, v)+\frac{N \lambda}{\mu} \rho(0, v)
$$

\section{Stationary distribution}

We can use Eqs. (3.8) and (3.9) to solve the continuous part of the stationary distribution of the system with two customers $(N=2)$.

As a first step we can evaluate Eqs. (3.8) and (3.9) for $N=2$. Then we obtain

$$
\rho(1, v)=\frac{F}{\mu} \frac{\partial}{\partial v} \rho(0, v)+\frac{2 \lambda}{\mu} \rho(0, v)
$$

and

$$
\begin{aligned}
\rho(n, v)= & \frac{(F-(n-1) f)}{n \mu} \frac{\partial}{\partial v} \rho(n-1, v) \\
& +\frac{((3-n) \lambda+(n-1) \mu)}{n \mu} \rho(n-1, v) \\
& -\frac{(4-n) \lambda}{n \mu} \rho(n-2, v),
\end{aligned}
$$

for $n=N=2$. Then, we obtain

$$
\rho(2, v)=\frac{(F-f)}{2 \mu} \frac{\partial}{\partial v} \rho(1, v)+\frac{(\lambda+\mu)}{2 \mu} \rho(1, v)-\frac{\lambda}{\mu} \rho(0, v) .
$$

So far, we have from Eq. (4.1) an expression for $\rho(1, v)$ in terms of $\rho(0, v)$. If we use this expression into Eq. (4.3) we can also obtain an expression of $\rho(2, v)$ in terms of $\rho(0, v)$ as follows

$$
\rho(2, v)=\frac{F(F-f)}{2 \mu^{2}} \frac{\partial^{2}}{\partial v^{2}} \rho(0, v)+\frac{(3 F \lambda-2 f \lambda+\mu F)}{2 \mu^{2}} \frac{\partial}{\partial v} \rho(0, v)+\frac{\lambda^{2}}{\mu^{2}} \rho(0, v) .
$$


Then, we can use Eq. (2.26) and evaluate expressions (4.1) and (4.4) for $\rho(1, v)$ and $\rho(2, v)$. We obtain

$$
\begin{gathered}
\frac{F(F-f)(F-2 f)}{2 \mu^{2}} \frac{\partial^{2}}{\partial v^{2}} \rho(0, v) \\
+\left(\frac{F(F-f)}{\mu}+\frac{(F-f)(F-2 f) \lambda}{\mu^{2}}+\frac{F(F-2 f)(\lambda+\mu)}{2 \mu^{2}}\right) \frac{\partial}{\partial v} \rho(0, v) \\
+\left(F+\frac{2 \lambda(F-f)}{\mu}+\frac{(F-2 f)(\lambda+\mu) \lambda}{\mu^{2}}-\frac{(F-2 f) \lambda}{\mu}\right) \rho(0, v)=0 .
\end{gathered}
$$

If we solve this equation we get the following result:

$$
\rho(0, v)=C_{01} \exp \left\{-2 \frac{F(\lambda+\mu)-2 f \lambda}{F(F-2 f)} v\right\}+C_{02} \exp \left\{\frac{(\lambda+\mu)}{F-f} v\right\} .
$$

If we consider $F(\lambda+\mu)-2 f \lambda=0$ and we solve for $F$, we obtain

$$
F=\frac{2 f \lambda}{\lambda+\mu}
$$

which is a consistent condition for systems with $N$ customers. Actually, this condition can also be constructed if we make $F$ equal to the expected average demand of a two-customer system. We start considering the long-term proportion of time that one customer is active, i.e., $\frac{\lambda}{\lambda+\mu}$. If we multiply this proportion by $f$ then we obtain the long-term average customer demand. Let us remember that each customer process is independent. Then, if we make $F$ equal to the average demand of the two-customer system, we have $F=\frac{2 f \lambda}{\lambda+\mu}$, which could be considered as an optimizing condition.

By considering condition (4.7) in Eq. (4.5) we can express this equation in the following form:

$$
\frac{\partial^{2}}{\partial v^{2}} \rho(0, v)+\frac{(\lambda+\mu)^{2}}{f(\lambda-\mu)} \frac{\partial}{\partial v} \rho(0, v)=0,
$$

which has the following solution:

$$
\rho(0, v)=C_{01}+C_{02} \exp \left\{-\frac{(\lambda+\mu)^{2}}{f(\lambda-\mu)} v\right\} .
$$

If we look at Eqs. (4.1) and (4.4) it is not difficult to see that

$$
\begin{aligned}
& \rho(1, v)=C_{11}+C_{12} \exp \left\{-\frac{(\lambda+\mu)^{2}}{f(\lambda-\mu)} v\right\}, \\
& \rho(2, v)=C_{21}+C_{22} \exp \left\{-\frac{(\lambda+\mu)^{2}}{f(\lambda-\mu)} v\right\} .
\end{aligned}
$$


Now, we can think again about the system with three customers. If we recall Eqs. (3.8) and (3.9), and we evaluate them for $N=3$. Then, we obtain

$$
\rho(1, v)=\frac{F}{\mu} \frac{\partial}{\partial v} \rho(0, v)+\frac{3 \lambda}{\mu} \rho(0, v)
$$

and

$$
\begin{aligned}
\rho(n, v)= & \frac{F-(n-1) f}{n \mu} \frac{\partial}{\partial v} \rho(n-1, v)+\frac{(4-n) \lambda+(n-1) \mu}{n \mu} \rho(n-1, v) \\
& -\frac{(5-n) \lambda}{n \mu} \rho(n-2, v)
\end{aligned}
$$

for $1<n \leq N$. Then we may evaluate this equation for $n=2$ and $n=3$ giving

$$
\rho(2, v)=\frac{(F-f)}{2 \mu} \frac{\partial}{\partial v} \rho(1, v)+\frac{2 \lambda+\mu}{2 \mu} \rho(1, v)+\frac{3 \lambda}{2 \mu} \rho(0, v)
$$

and

$$
\rho(3, v)=\frac{(F-2 f)}{3 \mu} \frac{\partial}{\partial v} \rho(2, v)+\frac{\lambda+2 \mu}{3 \mu} \rho(2, v)+\frac{2 \lambda}{3 \mu} \rho(1, v) .
$$

It is not difficult to prove that, as an extension of Eq. (2.26), for the two-customer system, the following equality holds for the three-customer system

$$
F \rho(0, v)+(F-f) \rho(1, v)+(F-2 f) \rho(2, v)+(F-3 f) \rho(3, v)=0 .
$$

Now, we can use expressions (4.11), (4.13) and (4.14) in Eq. (4.15) just as in the case for a two-customer system and we can obtain an expression for $\rho(0, v)$. If we use the condition

$$
F=\frac{3 f \lambda}{\lambda+\mu}
$$

which resembles condition (4.7), then we can express Eq. (4.15) in the following form:

$f^{2}(2 \lambda-\mu)(\lambda-2 \mu) \frac{\partial^{3}}{\partial v^{3}} \rho(0, v)+4 f(\lambda-\mu)(\lambda+\mu)^{2} \frac{\partial^{2}}{\partial v^{2}} \rho(0, v)+2(\lambda+\mu)^{4} \frac{\partial}{\partial v} \rho(0, v)=0$.

Eq. (4.16) can be constructed by making $F$ equal to the expected average demand of the three-customer system in the same way we constructed Eq. (4.7) for the twocustomer system. Also, we may find Eq. (4.16) as a condition to eliminate one of the roots in the equation for $\rho(0, v)$, just as we found Eq. (4.7). Conditions like these are present ahead in equations for systems with a larger number of customers.

It is not difficult to see that the solution for differential equation (4.17) is of the following manner: 


$$
\begin{aligned}
\rho(0, v)= & C_{01}+C_{02} \exp \left\{-\frac{(2 \lambda-2 \mu+\sqrt{2 \lambda \mu})(\lambda+\mu)^{2}}{(2 \lambda-\mu)(\lambda-2 \mu) f} v\right\} \\
& +C_{03} \exp \left\{-\frac{(2 \lambda-2 \mu-\sqrt{2 \lambda \mu})(\lambda+\mu)^{2}}{(2 \lambda-\mu)(\lambda-2 \mu) f} v\right\} .
\end{aligned}
$$

Therefore, solutions for $\rho(1, v), \rho(2, v)$ and $\rho(3, v)$ take the general form of $\rho(0, v)$

$$
\begin{aligned}
\rho(i, v)= & C_{i 1}+C_{i 2} \exp \left\{-\frac{(2 \lambda-2 \mu+\sqrt{2 \lambda \mu})(\lambda+\mu)^{2}}{(2 \lambda-\mu)(\lambda-2 \mu) f} v\right\} \\
& +C_{i 3} \exp \left\{-\frac{(2 \lambda-2 \mu-\sqrt{2 \lambda \mu})(\lambda+\mu)^{2}}{(2 \lambda-\mu)(\lambda-2 \mu) f} v\right\}
\end{aligned}
$$

for $i=0,1,2,3$.

Let us focus on equations for $\rho(0, v)$, which lead to the general form of the rest of the continuous part of the stationary distribution.

We can use Eqs. (3.8), (3.9) along with the $N$-general form equation

$$
\sum_{n=0}^{N}(F-n f) \rho(n, v)=0,
$$

that comes from the generalization of Eqs. (2.26) and (4.15), and the condition

$$
F=\frac{N f \lambda}{\lambda+\mu}
$$

that comes from the generalization of conditions (4.7) and (4.16), to find the set of equations for $N=1,4,5,6,7,8$ just as it is shown in Table 1 .

If we look at the equations in Table 1, it is not difficult to realize the pattern governing them. It can be seen that there is a difference between equations for even and odd $N$ according to factor

$$
\left(\frac{\partial}{\partial v}+\frac{(\lambda+\mu)^{2}}{(\lambda-\mu) f}\right)
$$

The rest of the equations follow the same pattern for every $N$. Then, we can write a tentative general equation for a system with any $N>2$ customers.

For odd $N>2$ we may say that 


\begin{tabular}{|c|c|}
\hline$N=1$ & $\frac{\partial}{\partial v} \rho(0, v)=0$ \\
\hline$N=2$ & $\left(\frac{\partial}{\partial v}+\frac{(\lambda+\mu)^{2}}{(\lambda-\mu) f}\right) \frac{\partial}{\partial v} \rho(0, v)=0$ \\
\hline$N=3$ & $\begin{array}{l}\left(f^{2}(2 \lambda-\mu)(\lambda-2 \mu) \frac{\partial^{2}}{\partial v^{2}}+4 f(\lambda-\mu)(\lambda+\mu)^{2} \frac{\partial}{\partial v}+2(\lambda+\mu)^{4}\right) \\
\times \frac{\partial}{\partial v} \rho(0, v)=0\end{array}$ \\
\hline$N=4$ & $\begin{array}{l}\left(f^{2}(3 \lambda-\mu)(\lambda-3 \mu) \frac{\partial^{2}}{\partial v^{2}}+6 f(\lambda-\mu)(\lambda+\mu)^{2} \frac{\partial}{\partial v}+3(\lambda+\mu)^{4}\right) \\
\times\left(\frac{\partial}{\partial v}+\frac{(\lambda+\mu)^{2}}{(\lambda-\mu) f}\right) \frac{\partial}{\partial v} \rho(0, v)=0\end{array}$ \\
\hline$N=5$ & $\begin{array}{l}\left(f^{2}(4 \lambda-\mu)(\lambda-4 \mu) \frac{\partial^{2}}{\partial v^{2}}+8 f(\lambda-\mu)(\lambda+\mu)^{2} \frac{\partial}{\partial v}+4(\lambda+\mu)^{4}\right) \\
\times\left(f^{2}(3 \lambda-2 \mu)(2 \lambda-3 \mu) \frac{\partial^{2}}{\partial v^{2}}+12 f(\lambda-\mu)(\lambda+\mu)^{2} \frac{\partial}{\partial v}+6(\lambda+\mu)^{4}\right) \\
\times \frac{\partial}{\partial v} \rho(0, v)=0\end{array}$ \\
\hline$N=6$ & $\begin{array}{l}\left(f^{2}(5 \lambda-\mu)(\lambda-5 \mu) \frac{\partial^{2}}{\partial v^{2}}+10 f(\lambda-\mu)(\lambda+\mu)^{2} \frac{\partial}{\partial v}+5(\lambda+\mu)^{4}\right) \\
\times\left(f^{2}(4 \lambda-2 \mu)(2 \lambda-4 \mu) \frac{\partial^{2}}{\partial v^{2}}+16 f(\lambda-\mu)(\lambda+\mu)^{2} \frac{\partial}{\partial v}+8(\lambda+\mu)^{4}\right) \\
\times\left(\frac{\partial}{\partial v}+\frac{(\lambda+\mu)^{2}}{(\lambda-\mu) f}\right) \frac{\partial}{\partial v} \rho(0, v)=0\end{array}$ \\
\hline$N=7$ & $\begin{array}{l}\left(f^{2}(6 \lambda-\mu)(\lambda-6 \mu) \frac{\partial^{2}}{\partial v^{2}}+12 f(\lambda-\mu)(\lambda+\mu)^{2} \frac{\partial}{\partial v}+6(\lambda+\mu)^{4}\right) \\
\times\left(f^{2}(5 \lambda-2 \mu)(2 \lambda-5 \mu) \frac{\partial^{2}}{\partial v^{2}}+20 f(\lambda-\mu)(\lambda+\mu)^{2} \frac{\partial}{\partial v}+10(\lambda+\mu)^{4}\right) \\
\times\left(f^{2}(4 \lambda-3 \mu)(3 \lambda-4 \mu) \frac{\partial^{2}}{\partial v^{2}}+24 f(\lambda-\mu)(\lambda+\mu)^{2} \frac{\partial}{\partial v}+12(\lambda+\mu)^{4}\right) \\
\times \frac{\partial}{\partial v} \rho(0, v)=0\end{array}$ \\
\hline$N=8$ & $\begin{array}{l}\left(f^{2}(7 \lambda-\mu)(\lambda-7 \mu) \frac{\partial^{2}}{\partial v^{2}}+14 f(\lambda-\mu)(\lambda+\mu)^{2} \frac{\partial}{\partial v}+7(\lambda+\mu)^{4}\right) \\
\times\left(f^{2}(6 \lambda-2 \mu)(2 \lambda-6 \mu) \frac{\partial^{2}}{\partial v^{2}}+24 f(\lambda-\mu)(\lambda+\mu)^{2} \frac{\partial}{\partial v}+12(\lambda+\mu)^{4}\right) \\
\times\left(f^{2}(5 \lambda-3 \mu)(3 \lambda-5 \mu) \frac{\partial^{2}}{\partial v^{2}}+30 f(\lambda-\mu)(\lambda+\mu)^{2} \frac{\partial}{\partial v}+15(\lambda+\mu)^{4}\right) \\
\times\left(\frac{\partial}{\partial v}+\frac{(\lambda+\mu)^{2}}{(\lambda-\mu) f}\right) \frac{\partial}{\partial v} \rho(0, v)=0\end{array}$ \\
\hline
\end{tabular}

Table 1. $\rho(0, v)$ equations for $N=1,2,3,4,5,6,7,8$ 


$$
\begin{aligned}
\left(\prod _ { n = 1 } ^ { ( N - 1 ) / 2 } \left(f^{2}((N-n) \lambda-n \mu)(n \lambda-(N-n) \mu) \frac{\partial^{2}}{\partial v^{2}}\right.\right. & \\
+2(N-n) n f(\lambda-\mu)(\lambda+\mu)^{2} \frac{\partial}{\partial v} & \\
\left.\left.+(N-n) n(\lambda+\mu)^{4}\right)\right) & \frac{\partial}{\partial v} \rho(0, v)=0 .
\end{aligned}
$$

For even $N>2$ we may say that

$$
\begin{aligned}
& \left(\prod _ { n = 1 } ^ { ( N / 2 ) - 1 } \left(f^{2}((N-n) \lambda-n \mu)(n \lambda-(N-n) \mu) \frac{\partial^{2}}{\partial v^{2}}\right.\right. \\
& \left.\left.+2(N-n) n f(\lambda-\mu)(\lambda+\mu)^{2} \frac{\partial}{\partial v}+(N-n) n(\lambda+\mu)^{4}\right)\right) \\
& \times\left(\frac{\partial}{\partial v}+\frac{(\lambda+\mu)^{2}}{(\lambda-\mu) f}\right) \frac{\partial}{\partial v} \rho(0, v)=0 .
\end{aligned}
$$

Now we can proceed to find a general solution for (4.22) and (4.23). As it can be seen, equations in Table 1 are factored in second order polynomials, therefore it is not difficult to find their roots. We obtain the general solutions as follows:

For odd $N>2$,

$$
\begin{aligned}
& \rho(i, v)=C_{i, 0}+\sum_{n=1}^{(N-1) / 2} C_{i, n} \\
& \times \exp \left\{-\frac{((N-n) n(\lambda-\mu)+(N-2 n) \sqrt{(N-n) n \lambda \mu})(\lambda+\mu)^{2}}{((N-n) \lambda-n \mu)(n \lambda-(N-n) \mu) f} v\right\} \\
& +\sum_{m=1}^{(N-1) / 2} C_{i, N-m} \\
& \times \exp \left\{-\frac{((N-m) m(\lambda-\mu)-(N-2 m) \sqrt{(N-m) m \lambda \mu})(\lambda+\mu)^{2}}{((N-m) \lambda-m \mu)(m \lambda-(N-m) \mu) f} v\right\} .
\end{aligned}
$$

For even $N \geq 2$, 


$$
\begin{aligned}
& \rho(i, v)=C_{i, 0}+C_{i, N / 2} \exp \left\{-\frac{(\lambda+\mu)^{2}}{(\lambda-\mu) f} v\right\}+\sum_{n=1}^{(N / 2)-1} C_{i, n} \\
& \times \exp \left\{-\frac{((N-n) n(\lambda-\mu)+(N-2 n) \sqrt{(N-n) n \lambda \mu})(\lambda+\mu)^{2}}{((N-n) \lambda-n \mu)(n \lambda-(N-n) \mu) f} v\right\} \\
& +\sum_{m=1}^{(N / 2)-1} C_{i, N-m} \\
& \times \exp \left\{-\frac{((N-m) m(\lambda-\mu)-(N-2 m) \sqrt{(N-m) m \lambda \mu})(\lambda+\mu)^{2}}{((N-m) \lambda-m \mu)(m \lambda-(N-m) \mu) f} v .\right.
\end{aligned}
$$

As we know, these solutions are not only valid for $\rho(0, v)$, but also for the general form of the rest of the stationary distribution. Nevertheless we may just consider the constants from $\rho(0, v)$ as $C_{0, n} \Leftrightarrow C_{n}, n \in\{0,1, \ldots, N-1\}$ in order to simplify the solution and we may express the rest of the distributions $\rho(i, v)$ in terms of $\rho(0, v)$ using Eqs. (3.8) and (3.9).

The analysis of the properties of the process $\xi^{(N)}(t)$ leads up to the conclusion that, for the case $a f<F<b f$, the stationary distribution $\rho$ has atoms at the points $(b, 0),(b+1,0), \ldots,(N, 0)$, and at the points $(0, V),(1, V), \ldots,(a, V)$. We denote them as $\rho[b, 0], \rho[b+1,0], \ldots, \rho[N, 0]$ and $\rho[0, V], \rho[1, V], \ldots, \rho[a, V]$.

We get from the discrete part of Eq. (3.4) the following expressions.

$$
\begin{gathered}
{\left[\begin{array}{cccccc}
F & 0 & 0 & \ldots & 0 & 0 \\
0 & F-f & 0 & \ldots & 0 & 0 \\
0 & 0 & F-2 f & \ldots & 0 & 0 \\
\vdots & \vdots & \vdots & \vdots & \vdots & \vdots \\
0 & 0 & 0 & \ldots & 0 & 0 \\
0 & 0 & 0 & \ldots & F-(N-1) f & 0 \\
0 & 0 & 0 & \ldots & 0 & F-N f
\end{array}\right]\left[\begin{array}{c}
\rho(0,0+) \\
\rho(1,0+) \\
\rho(2,0+) \\
\vdots \\
\rho(N-2,0+) \\
\rho(N-1,0+) \\
\rho(N, 0+)
\end{array}\right]} \\
=Q^{(N) T} \mathbf{R}(0),
\end{gathered}
$$


where $\mathbf{R}(0)=\left[\begin{array}{c}0 \\ \vdots \\ 0 \\ \rho[b, 0] \\ \rho[b+1,0] \\ \vdots \\ \rho[N, 0]\end{array}\right]$. Also,

$\left[\begin{array}{cccccc}F & 0 & 0 & \ldots & 0 & 0 \\ 0 & F-f & 0 & \ldots & 0 & 0 \\ 0 & 0 & F-2 f & \ldots & 0 & 0 \\ \vdots & \vdots & \vdots & \vdots & \vdots & \vdots \\ 0 & 0 & 0 & \ldots & 0 & 0 \\ 0 & 0 & 0 & \ldots & F-(N-1) f & 0 \\ 0 & 0 & 0 & \ldots & 0 & F-N f\end{array}\right]\left[\begin{array}{c}\rho(0, V-) \\ \rho(1, V-) \\ \rho(2, V-) \\ \vdots \\ \rho(N-2, V-) \\ \rho(N-1, V-) \\ \rho(N, V-)\end{array}\right]$
$=-Q^{(N) T} \mathbf{R}(V)$,

where $\mathbf{R}(V)=\left[\begin{array}{c}\rho[0, V] \\ \rho[1, V] \\ \vdots \\ \rho[a, V] \\ 0 \\ \vdots \\ 0\end{array}\right]$.

If we use $b=a+1$, that is, $a<F<a+1$ is easy to see from Eq. (4.26) that

$$
\left\{\begin{aligned}
\rho(0,0+) & =0, \\
\rho(1,0+) & =0, \\
\vdots & \\
\rho(a-1,0+) & =0 .
\end{aligned}\right.
$$

Also, from Eq. (4.27) we obtain that 


$$
\left\{\begin{aligned}
\rho(a+2, V-) & =0 \\
\rho(a+3, V-) & =0 \\
\vdots & \\
\rho(N, V-) & =0 .
\end{aligned}\right.
$$

It is not difficult to use Eqs. (4.28) and (4.29) to obtain expressions for the constants $C_{n}, n \in\{1,2, \ldots, N-1\}$.

Also, by using expressions in Eq. (4.26) not equal to zero we may obtain expressions for the atoms in $\mathbf{R}(0)$ in terms of the continuous part $\rho(\theta, 0+)$. In the same way we may use (4.27) to obtain expressions for the atoms in $\mathbf{R}(V)$ in terms of the continuous part $\rho(\theta, V-)$.

After that it is not difficult to calculate constant $C_{0}$ using the normalization condition

$$
\int_{\mathbb{W}^{(\mathbb{N})}} \rho(w) \mathrm{d} w=1 .
$$

That completes the calculation of the stationary distribution $\rho$ of the system.

\section{Conclusions}

It is possible to use a phase merging algorithm to reduce a semi-Markov process to an approximated Markov process. Once this is done, it is possible to find some closedform expression for the stationary probability distribution of the system.

We have found that the problem of the single buffer with $N$ equal customers connected to it has a general solution for the stationary probability distribution of the amount of stored information. We found this general solution considering only one condition that could be considered as an optimizing condition. We constructed this condition so that the stream of information is equal to the expected average demand of the system, and it is also present as one of the roots of the general equation to be solved. By having this condition, the general equation is simplified so that the solution may present one less exponential term.

Even though it was mentioned as part of the system functionality that the main stream $F$ turns off when the buffer reaches its maximum capacity, the results presented here also match to those of a system with an overflowed buffer. That is, a system where the main stream $F$ is always on and when the buffer reaches its maximum capacity some data may be thrown away.

Acknowledgments. We thank Tecnológico de Monterrey, Campus Monterrey, through the Research Chair in Telecommunications for the support provided in the development of this work. 


\section{References}

1. A. Pogorui, and A. F. Turbin, Estimation of Stationary Efficiency of a Production Line with Two Unreliable Aggregates. Cybernetics and Systems Analysis, 38, No. 6, pp. 823$829,2002$.

2. J. A. Korlat, V. N. Kuznetsov, M. M. Novikov, and A. F. Turbin, Semi-Markov Models of Restorable Systems and Queueing Systems (in Russian). Shtiintsa, Kishinev, 1991.

3. V. S. Korolyuk, and A. V. Swishchunk, Semi-Markov Random Evolutions. Kluwer Academic Publishers, 1995.

4. V. S. Korolyuk, and V. V. Korolyuk, Stochastic models of systems. Kluwer Academic Publishers, 1998.

5. V. S. Korolyuk, and A. F. Turbin, Mathematical Foundations of the State Lumping of Large Systems. Kluwer Academic Publishers, 1994.

6. J. Wijngaard, The Effect of Interstage Buffer Storage on the Output of Two Unreliable Production Units in Series, with Different Production Rates. AIIE Transactions, 11 (1979), $42-47$.

7. J. P. Malathronas, J. D. Perkins, and R. L. Smith, The Availability of a System of Two Unreliable Machines Connected by an Intermediate Storage Tank. IIE Transactions, 15 (1983), 195-201.

8. V. G. Kulkarni, Fluid Models for Single Buffer Systems in Frontiers in queueing. Probability and Stochastic Series, CRC, Boca Raton, FL. Editor J.H. Dshalalow, pp. 321-336, 1997.

9. M. Reed, and B. Simon, Methods of Modern Mathematical Physics, Vol. 1, Academic Press, 1972.

Received 2 June, 2006; revised 14 April, 2007

\section{Author information}

R. D. Rodríguez-Said, Monterrey Institute of Technology (ITESM), México. Email: rd.rodriguez.said@itesm.mx

A. A. Pogorui, Monterrey Institute of Technology (ITESM), México.

Email: pogorui@itesm.mx

R. M. Rodríguez-Dagnino, Monterrey Institute of Technology (ITESM), Sucursal de correos "J" C.P. 64849, Monterrey, N.L., México.

Email: rmrodrig@itesm.mx 